\title{
3D Ultrasound Image Acquisition Using a Magneto-optic Hybrid Sensor for Laparoscopic Surgery
}

\author{
Yoshinobu Sato $^{1}$, Masaki Miyamoto ${ }^{1}$, Masahiko Nakamoto ${ }^{1}$, \\ Yoshikazu Nakajima ${ }^{1}$, Mitsuo Shimada ${ }^{2}$, Makoto Hashizume ${ }^{3}$, and \\ Shinichi Tamura ${ }^{1}$ \\ 1 Division of Interdisciplinary Image Analysis \\ Osaka University Graduate School of Medicine \\ 2 Department of Surgery II \\ 3 Department of Disaster and Emergency Medicine \\ Graduate School of Medical Science, Kyushu University \\ yoshi@image.med.osaka-u.ac.jp, http://www.image.med.osaka-u.ac.jp/yoshi
}

\begin{abstract}
A 3D ultrasound system suitable for laparoscopic surgery using a novel configuration of a magneto-optic hybrid 3D sensor is reported. A miniature magnetic sensor with five degrees of freedom (5D) is combined with an optical sensor outside the body to perform $6 \mathrm{D}$ sensing of the flexible probe in the abdominal cavity. The accuracy of the system is evaluated in comparison with a conventional $3 \mathrm{D}$ ultrasound system.
\end{abstract}

\section{Introduction}

In liver surgery, 3D ultrasound (3D-US) is a useful intraoperative imaging modality to assist the surgeon in recognizing spatial relationships between tumors and vessels. Although use of the laparoscope is becoming common as a minimally invasive procedure, using it in combination with 3D-US has not been reported. We have developed a 3D-US system that can be utilized in laparoscopic liver surgery. The tip of the ultrasound probe can be flexibly moved in the abdominal cavity. To measure six degrees of freedom (6D) for the position and orientation of the flexible probe tip, a novel magneto-optic hybridization configuration is employed. Conventional 6D magnetic sensors are too large to be inserted into the abdominal cavity without additional incision, while optical sensors suffer from the line of sight constraint. To circumvent these drawbacks, we combine a miniature magnetic sensor only $1 \mathrm{~mm}$ diameter which can be inserted into the abdomen without an additional incision but which measures only five degrees of freedom (5D), with an optical sensor outside the abdomen to localize the probe tip with $6 \mathrm{D}$ in the abdominal cavity.

\section{Methods}

The complete system is depicted diagramatically in Fig. 1(a). Registration of the coordinate systems of the magnetic and optical sensors is performed based on our 
previous framework of magneto-optic hybridization [1]. Figure 1(b) shows the ultrasound probe (Aloka, Tokyo) that we employ in laparoscopic surgery. The rigid body attached outside the abdomen defines the probe-centered coordinate system (PCS). The probe tip is flexible but its motion is restricted within a plane (Fig.1(c)). In the preoperative calibration stage, we determine the plane of the probe tip motion in PCS by gathering 5D measurements of the tip in various arrangements, thereby obtaining the fixed spatial relationship between the rigid body and the plane of the probe tip motion. In the intraoperative stage, the probe tip plane is estimated by combining the position and orientation of PCS measured using an optical sensor and the fixed relationship obtained in the calibration stage. The position and orientation within the probe tip plane are estimated using a miniature magnetic sensor (3D translation and 2D rotation). In this way, $6 \mathrm{D}$ parameters of the probe tip position and orientation are obtained, and the tip-centered coordinate system (TCS) is defined. Finally, the ultrasound image coordinate system (UICS) is determined by combining the relationship of TCS and UICS determined in the preoperative calibration stage [2].

\section{Experimental Results}

Experiments were performed to evaluate the accuracy of the system. Since our magnetic tracking system of choice, Aurora (Northern Digital Inc.), was not operational at the time we carried out the experiments, we provisionally substituted Fastrak (Polhemus Inc.) as the magnetic sensor and utilized only its $5 \mathrm{D}$ parameters so that it functioned in a similar manner to Aurora. Polaris (Northern Digital Inc.) was employed as the optical sensor. The 3D position of a phantom tip in a water bath (Fig.1(d)) was measured using the following three methods: (i) The Polaris pen-probe digitizer (Let $\mathbf{x}_{p}$ be its measurements).

(ii) The proposed 3D-US system using a magneto-optic hybrid sensor $\left(\mathbf{x}_{h}\right)$. (iii) A conventional 3D-US system with 6D magnetic sensor (Fastrak) directly attached to the probe tip $\left(\mathbf{x}_{m}\right)$. The accuracies of the proposed and conventional 3D-US systems were evaluated by regarding the measurements of the Polaris pen-probe digitizer as the gold standard, that is, the errors were defined as follows: $\Delta \mathbf{x}_{h}=\left|\mathbf{x}_{p}-\mathbf{x}_{h}\right|$ and $\Delta \mathbf{x}_{m}=\left|\mathbf{x}_{p}-\mathbf{x}_{m}\right|$. Thirty measurements for the 3D position of the phantom tip were obtained with various probe tip arrangements. Table 1 shows the average (bias) and standard deviation (SD) of the errors. The accuracy of the proposed system, which is suitable for laparoscopic surgery, was comparable to that of the conventional one, which is difficult to use for that purpose.

\section{Conclusion}

We have described a 3D ultrasound system that employs a magneto-optic hybrid sensor and is suitable for use in laparoscopic surgery. The accuracy of the system was comparable to that of the conventional one, which is not suited to laparoscopic surgery. We will shortly replace the conventional magnetic sensor 
Table 1. Accuracy evaluation. $\left(\delta_{x}, \delta_{y}, \delta_{z}\right)$ and $\left(\sigma_{x}, \sigma_{y}, \sigma_{z}\right)$ respectively denote bias and standard deviation (SD) with respect to each axis; $\sigma_{D}=\left(\sigma_{x}^{2}+\sigma_{y}^{2}+\right.$ $\left.\sigma_{z}^{2}\right)^{0.5}$.

\begin{tabular}{c|c|c} 
& Bias: $\left(\delta_{x}, \delta_{y}, \delta_{z}\right)(\mathrm{mm})$ & $\mathrm{SD}:\left(\sigma_{x}, \sigma_{y}, \sigma_{z}\right), \sigma_{D}(\mathrm{~mm})$ \\
\hline Proposed 3D-US & $(0.40,0.31,0.71)$ & $(0.44,0.84,0.53), 1.08$ \\
\hline Conventional 3D-US & $(0.26,1.38,0.82)$ & $(0.42,0.99,0.47), 1.18$ \\
\hline
\end{tabular}

(Fastrak) with a miniature one (Aurora) for 5D sensing in the abdominal cavity. We are planning to extend the system so as to realize augmented reality visualization, which superimposes 3D ultrasound images onto laparoscopic images [2]. Acknowledgement: This work was partly supported by JSPS Research for the Future Program JSPS-RFTF99I00903 and JSPS Grant-in-Aid for Scientific Research (B)(2) 12558033.

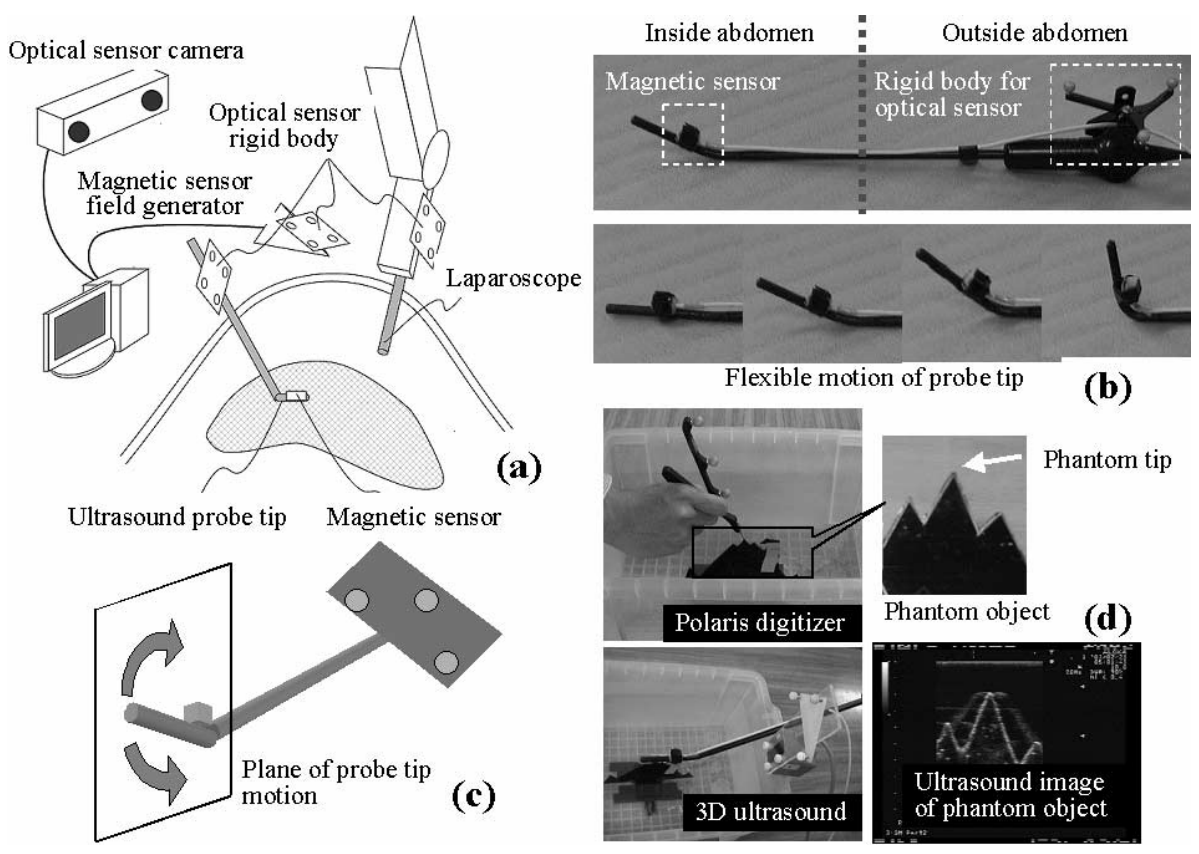

Fig. 1. 3D ultrasound system for laparoscopic surgery. (a) System diagram. (b) Ultrasound probe for laparoscopic surgery. (c) Plane of probe tip motion (probe tip plane). (d) Laboratory experiments for accuracy evaluation.

\section{References}

1. Nakamoto M et al., Magneto-optic hybrid 3-D sensor for surgical navigation, LNCS, 1935 (Proc. MICCAI 2000), 839-848 (2000).

2. Sato $\mathrm{Y}$ et al., Image guidance of breast cancer surgery using $3 \mathrm{D}$ ultrasound images and augmented reality visualization, IEEE-TMI, 17(5), 681-693 (1998). 